\title{
Systems Dynamics Modeling of Pandemic Influenza for Strategic Policy Development: a Simulation-Based Analysis of the COVID-19 Case
}

\author{
Charlle Sy ${ }^{1,2} \cdot$ Phoebe Mae Ching ${ }^{3} \cdot$ Jayne Lois San Juan ${ }^{1,2}$ (D) Ezekiel Bernardo ${ }^{1} \cdot$ Angelimarie Miguel $^{1}$. \\ Andres Philip Mayol ${ }^{2,4}$. Alvin Culaba ${ }^{2,4}$ - Aristotle Ubando ${ }^{2,4}$. Jose Edgar Mutuc ${ }^{1,2}$
}

Received: 16 September 2020 / Revised: 27 December 2020 / Accepted: 1 January 2021 / Published online: 28 January 2021

(C) The Author(s), under exclusive licence to Springer Nature Singapore Pte Ltd. part of Springer Nature 2021

\begin{abstract}
The novel coronavirus disease 2019 (COVID-19) is a truly wicked problem which has remained a stubborn issue plaguing multiple countries worldwide. The continuously increasing number of infections and deaths has driven several countries to implement control and response strategies including community lockdowns, physical distancing, and travel bans with different levels of success. However, a disease outbreak and the corresponding policies can cause disastrous economic consequences due to business closures and risk minimization behaviors. This paper develops a system dynamics framework of a disease outbreak system covering various policies to evaluate their effectiveness in mitigating transmission and the resulting economic burden. The system dynamics modeling approach captures the relationships, feedbacks, and delays in such a system, revealing meaningful insights on the dynamics of several response strategies.
\end{abstract}

Keywords COVID-19 $\cdot$ Disease outbreak $\cdot$ Response strategies $\cdot$ Policy recommendation $\cdot$ Impact assessment

\section{Introduction}

The outbreak of coronavirus disease 19 (COVID-19) has severely affected several countries. The respective healthcare sectors have become overburdened with demands for testing, care and treatment. Aside from a strain in their capacity, healthcare workers are also reported to have increased prevalence of mental health disorders, typically correlated to

This article is part of the Topical Collection on COVID-19: optimization strategies to combat pandemic.

Charlle Sy

charlle.sy@dlsu.edu.ph

1 Industrial Engineering Department, De La Salle University, 2401 Taft Avenue, 0922 Manila, Philippines

2 Center for Engineering and Sustainable Development Research, De La Salle University, 2401 Taft Avenue, 0922 Manila, Philippines

3 Department of Industrial Engineering and Decision Analytics, Hong Kong University of Science and Technology, Clear Water Bay, Kowloon, Hong Kong

4 Mechanical Engineering Department, De La Salle University, 2401 Taft Avenue, 0922 Manila, Philippines healthcare workers who have frequent direct contact with infected patients, as well as access to personal protective equipment (Pappa et al. 2020). This is supported by a study performed by Zhang et al. (2020) on healthcare staff in Iran detailing declining physical and mental healths and increasing anxiety, depression, and distress. Mounting anxieties on the availability of supplies have also resulted in civil unrest and individual trauma (Webster et al. 2020). Moreover, the extended closure of establishments and impairment of business operations will have lasting negative impact on various aspects of the economy (Hart and Halden 2020). Many businesses are negatively hit by this crisis and are trying to devise strategic responses to minimize the damage, as well as quicken their recovery (Wenzel et al. 2020). The gravity of these problems can be minimized through policy management. The mode, duration, and level of enforcement of policies employed to handle can determine the trend of infection, as well as how long a country suffers from the consequences following the outbreak.

As a disease, the symptoms of coronavirus include respiratory ailments, fever, and fatigue. It is related to two viruses which resulted in nationwide outbreaks in the past, namely, the severe acute respiratory syndrome (SARS) and the Middle East respiratory syndrome (MERS) (Yang et al. 2020b). From some 
clinical trials, it has been found that some forms of treatment against the aforementioned diseases are potentially effective against COVID-19, namely, type 1 interferons (Sallard et al. 2020), chloroquine, and hydroxychloroquine (Singh et al. 2020). These are currently being implemented in hospitals, although the exact parameters for giving treatment may still vary per location. There are currently attempts to develop vaccines and additional treatments for COVID-19. However, the extensive testing required before these discoveries can be endorsed to the general public indicates that we cannot rely on a medical response to completely resolve COVID-19 (Le et al. 2020). Instead, the more efficient response is to "starve" the disease through policies that control disease transmission.

The COVID-19 outbreak is an unprecedented event in history. Knowledge on the infection trend would be helpful to policy-makers, in making investments and planning for future responses (Ejima and Nishiura 2018). However, there is no past data on COVID-19 to derive this trend from forecasting models. Although there have been outbreaks, albeit at a smaller scale, in the past, there are some unique aspects of the COVID-19 situation that can influence the behavior of infection. The limitations of using forecasting methods and the insights to gain in policy management are discussed in more detail in the following section.

Still, an analytical approach to policy development can be helpful in developing solutions that are efficient, are impactful, and can be logically proven. Such methods are also important because trial-and-error is not an option in the current situation. A mechanistic modeling approach, where causation is represented in equation form, can be a suitable alternative for data when it is not. Germann et al. (2019) support this argument and applied stochastic simulation modeling to evaluate various school dismissal policies in mitigating influenza pandemic transmissions. Similarly, Towers et al. (2011) utilized mechanistic modeling to study the impact of antiviral treatment programs on the size of seasonal pandemic influenza. Moreover, these have the advantage of being able to incorporate the impact of solutions. Thus, solutions can be simulated prior to implementation, and its parameters can be further optimized upon development.

The proposed study positions system dynamics (SD) as an efficient framework for policy development. It has been used extensively in the management of supply chains (Fontes and Freires 2018), technology diffusion, and even the development of government policies. SD was selected as the methodology of this study because of its flexibility. It allows for unquantifiable variables to be integrated in models, replacing data with feedback relationships. This makes it suitable in solution development or systems design for unprecedented situations. Alamerew and Brissaud (2020) utilized the SD approach to model the dynamics of cost, revenue, and regulatory decisions for the reverse logistics of electric vehicle batteries, while Cao et al. (2019) simulated various CO2 mitigations for a green electric-coal supply chain. SD has also been applied by Lei and Zhou (2020) to understand the dynamics of a reactiondiffusion system considering negative feedback and inhibition for technology diffusion. Similarly, Wang and Lai (2020) developed an SD model to evaluate the diffusion of various mobile payment platforms under varying platform design strategies. Kuo et al. (2019) developed a SD model to explore the potential of commercializing biofuels for vehicles in Taiwan with government subsidies. A sustainability assessment using SD modeling was performed by Espinoza et al. (2017) for various biodiesel government policies in Colombia. Zhao et al. (2017) investigated enterprises' compliance with a carbon reduction labelling policy given a combined scheme of government subsidies and economic penalties.

It is efficient as it relies less on computational exactness and volume of data, and more on simple causality and a deep understanding of the system at hand, to generate results (Forrester 1994). The strength of SD is its organization of factors into causal loops, to capitalize on delays in the correlations between factors, and specify the area and timing of implementation accordingly (Deng et al. 2020). The key advantage of SD in application to pandemics is its realistic ability to represent human behavior as "soft" variables in the model (Doyle and Ford 1998). With recent strategies being heavily reliant on human participation and social mobilization, policy models need a means to integrate this as a factor without making the model unbalanced in quantification. Overall, the framework offers a unique perspective on situations and opens an opportunity for the discovery of new solutions in response to COVID-19.

The general objective of this study is to develop policies to address the transmission of COVID-19 within a population through the system dynamics framework, extending the previous work by Sy et al. (2020) which considered only a simplified pandemic transmission and response system by giving focus on various configurations of community quarantine initiatives. Specifically, its objectives are:

- To identify and characterize the variables and dynamic relationships contributing to the transmission of COVID19 in a population

- To determine leverage points among the variables representing the COVID-19 transmission problem

- To define concrete policies based on the leverage points and their impact on transmission

The rest of this article is organized as follows: the "Literature Review" section presents a survey of relevant literature beginning with various modeling techniques adapted in the study of disease outbreaks such as pandemics and epidemics, and policies developed to curb the incidence of pandemics. The "Model Development" section discusses the SD model developed which extends the basic SEIR model and 
study by Sy et al. (2020) to capture the dynamics of social mobilization strategies, public health and hospital care systems, and the impact to the economy to the transmission of diseases, particularly considering COVID-19 in the Philippines as a case study as demonstrated in the "Simulation Results" section. The "Simulation Results" section also testifies to the sensitivity of the system to changes in key parameters that could be targeted through certain policies. These insights are summarized in the "Policy Insights/ Recommendations" section to provide an actionable guide to policy-makers. Lastly, conclusions and directions for future research are provided in the "Conclusions and Future Directions" section.

\section{Literature Review}

\section{Pandemic Modeling}

For pandemics, one of the primary parameters of interest is the trend of infection. Ejima and Nishiura (2018) applied the method of maximum likelihood estimates to predict the number of infected segmented by age, based on the age-dependent infection pattern. The study demonstrated a methodology for real-time predictions on infection; however, the final results showed that at least 5 months of data would be needed to make reliable predictions. Giovannelli et al. (2015) used epidemic curve modeling to predict hospital bed and ICU utilizations using information on hospitalization rate, ICU admission rate, and general practitioner care parameters. However, the accuracy of parameters was limited by the lack of robust data sources. In particular, the authors believed that information on vaccinations, treatment, and the influence of school closures could have improved the predictive capabilities of their model. Chintalapudi et al. (2020) used past information on infection, recovery, and death to predict the number of cases and recoveries in the coming periods. The seasonal ARIMA model developed by the aforementioned showed that the decrease in infection coincided with the lockdown period. However, similar to Giovannelli et al. (2015), the authors also felt that additional data, particularly on the contribution of healthcare availability, would improve the accuracy of the model. Su et al. (2019) used ARIMA and XGBoost to predict the percentage of influenza-like illness (\%ILI) in a population. The model used data on past \%ILI, weather, and Baidu searches as predictors. However, the authors concluded that further granularization was necessary to improve the accuracy of the model.

Applied to pandemics, the primary objective of forecasting models is to predict the number of infected cases. Infection is predicted over a period of time, which can support decisionmaking on prioritization, capacity planning, and resource allocation in response to the pandemic. Ideally, it would be possible to make predictions using historical data; however, it may be time before sufficient data is available (Ejima and Nishiura 2018). Most studies have employed variables external to infection to improve the accuracy of predictions. All forecasting studies that were reviewed cited the lack of granular data of the external variables as a barrier to accuracy. In some cases, there was no means to obtain data for a particular variable that the researchers felt would have strengthened their model (Giovannelli et al. 2015; Su et al. 2019; Chintalapudi et al. 2020). It is worthy of note that the lack of data for supporting variables and its impact on forecasting accuracy has been cited as early as 2009 , during the time of the A(H1N1) pandemic (Santos-Preciado et al. 2009). The dependence on substantial data for model development is a major weakness of this approach, particularly when the intention is to be able to use the model in real time for decision-making. Tang et al. (2020) aptly likens the forecasting of COVID-19 and its outcomes to stock-market forecasting, helpful, but requiring insurance to account for the variables that cannot be modeled with data.

Mechanistic modeling can be an alternative to data-driven models, by deriving the behavior of a target variable from its interactions with other variables in its environment. These interactions are represented as mathematical equations. For disease outbreaks, the susceptible infectious recovered (SIR) model is a common mechanistic modeling approach. Towers et al. (2011) developed a seasonal SIR model to derive the number of infected cases. This approach allowed for solutions to be simulated, particularly the impact of vaccines and treatment on which no data may be available yet. The authors indicated the possibility of a second peak in infection as a limitation of this modeling approach, to be improved in future studies. Li and Muldowney (1995), on the other hand, add a fourth category to the population, studying an SEIR model which extended SIR to capture fractions of the population that are susceptible (S), exposed (E), infectious (I), and recovered (R). Germann et al. (2019) used the so-called epidemiological forecasting model in deriving the volume of infected cases. The study was able to demonstrate the impact of school closures and identify optimal closure durations. Modeling can also be made specific to a solution. Sun et al. (2014) optimized the necessary travel distance for patients and resource efficiency of a community, by determining the allocation of additional resources to hospitals based on population density. Yang et al. (2020b) have applied the general equilibrium model in determining tourism demand under COVID-19, through an understanding of the parameters that influence tourism.

The mechanistic modeling approach nullifies the absence of data by modeling the interactions between factors, rather than deriving the relationship via regression techniques. It is particularly useful for solution development. The impact of solutions that have yet to be applied can be simulated for 
greater efficiency; this is helpful for assessing and validating the helpfulness of vaccines and treatment at different stages of the pandemic (Yang et al. 2020b; Sun et al. 2014; Towers et al. 2011; Culaba et al. 2019). Moreover the direct impact of solutions on the target variable can be modeled, to identify the specific parameters for implementing the solution (Sun et al. 2014; Germann et al. 2019).

Generally, the model representation of a system becomes more realistic as more sectors are involved. In this way, the limitations and consequences of implementing certain solutions can be considered. For most modeling approaches, involving a large number of variables is a computationally expensive endeavor. In system dynamics (SD) models, the physical exactness of the modeled interactions is relaxed, making it relatively efficient in that aspect. For this reason, system dynamics has been used extensively in policy development for situations involving multiple sectors, with their own dynamic relationships. Relaxing the physical exactness of the equations can put the validity of models into question. To address this, some studies have integrated SD with other modeling frameworks that can validate its results, particularly with particle filtering (Safarishahrbijari et al. 2017) and analytical hierarchy process (AHP) (Araz 2013). Such studies demonstrate that system dynamics can be further improved when coupled with other modeling frameworks.

On its own, system dynamics has advantages when applied in pandemic modeling. First, the absence of data on the current situation limits the accuracy of forecasts, and models based on actual cause-and-effect can represent the system more accurately. In forecasting changes to product demand during an influenza crisis, Thiel et al. (2014) showed that system dynamics could replicate behavior more robustly than forecasting models. In conjunction with this modeling style, an alternative to finding the most significant factor through statistical analysis can also be achieved through a what-if analysis of parameters, the results of which can be more meaningful and more straightforward for policy development. Vo and Thiel (2011) have used this approach to identify policies resulting in non-oscillatory behavior, implying that the problem has been resolved completely, without the possibility of recurrence. Ibarra-Vega (2020) assessed the effectiveness of lockdowns imposed on various levels of stringency and timeline in reducing contacts between the susceptible and infected population using a system dynamics model. Moreover, the problem can be assessed concurrently at different levels of depth, revealing more points of intervention for policy development. Tuckwell and Toubiana (2007) modeled infection dynamics at a micro-scale within the immune system, and at macro-scale between persons, demonstrating a capability that is not possible in forecasting models and highly complex in mechanistic models. Araz (2013) proposed a hybrid methodology combining AHP with system dynamics modeling to evaluate the impact of mitigation strategies, particularly various school closures policies, on disease progression. Nonetheless, several other factors come into play in this system, such as healthcare capacity strain and economic strain. Moreover, Currie et al. (2020) identify a set of issues realized during the COVID-19 pandemic that are particularly suited to be solved by various simulation techniques. These include decisions relevant to minimizing transmission through lockdowns and social distancing, managing healthcare and medical resources, and delivering care (i.e., vaccination, hospital admission, and impact to non-COVID patients).

\section{Policy Development for Pandemics}

\section{Social Mobilization}

Social mobilization prevents infection through individual behavior. These initiatives can range from social pressures to proclamations from government officials (Westerhoff et al. 2018). Yet, ultimately the choice of whether or not to participate remains on the individual.

In the case of COVID-19, the outcomes of social mobilization efforts include improved hygiene practices, wearing personal protective equipment, and social distancing without enforcement. These represent the willingness of members of a population to participate in preventing infection, which can remove roadblocks against the implementation of certain policies. In India, social mobilization improved the efficiency of vaccination programs against cholera (Gupta and Gupta 2020). This reduced resource expenditure on program enforcement and controls the spread of the disease among those who have yet to be vaccinated.

Aside from preventing infection, social mobilization can also prevent other problems associated with the pandemic. Mental disorders, such as trauma, depression, and anxiety related to COVID-19, along with the physical comorbidities of mental disorders, are perceived to be the next wave of the pandemic (Chevance et al. 2020). Holmes et al. (2020) likewise cite mental health science research to be one of the immediate research priorities under this COVID-19 pandemic. This poses a new burden on social workers and healthcare professionals. Exercising at home is being encouraged as a means to boost immunity and mental stability under lockdown (Simpson and Katsanis 2020). This can mitigate the extended effects of COVID-19 on a population, even after infection rate has come under control.

\section{Community Quarantine}

Quarantine is defined as the separation of individuals or groups with exposure to a contagious disease, in order to control infection. The extent of enforcement required in the implementation of quarantine can vary depending on necessity (Webster et al. 
2020). In some cases, it is sufficient to increase public knowledge, as in social mobilization; conversely, law enforcement may be required to ensure adherence.

The quarantine of populations as large and geographically dispersed as a city or country is defined as community quarantine. A modeling study on the Ebola outbreak showed that the appropriate levels of coverage and effectiveness contribute significantly to the effectiveness of disease control measures (Dénes and Gumel 2019). A sampling study on the COVID19 pandemic showed that the early implementation and strict enforcement of home quarantine helped infection, although improving capacity for early detection could further improve these numbers (Wang et al. 2020). Furthermore, there is evidence from statistics on COVID-19 that the trend of infection is dependent on the amount of quarantined and suspected cases, respectively (Tang et al. 2020).

The consequences of community quarantine on the economy will be more severe than those of social mobilization. Aside from this, there is a risk of long-term psychological effects. Post-traumatic stress symptoms resulting from the pandemic have been reported, with the stressors being frustration, boredom, and anxieties about infection, financial losses, and inadequate supplies (Brooks et al. 2020). Negative psychological consequences have already been observed on individuals who have expressed dissatisfaction with the quarantine measures (Zhu et al. 2020). In the past, it has proven helpful to enforcement for authorities to emphasize the altruistic connotations of quarantine, the potential benefit on public health, and the availability of supplies (Webster et al. 2020). For these reasons, the coverage of quarantine and the resources deployed in its implementation are a critical decision during the time of a pandemic.

\section{Treatment of Infected Persons}

Pandemics call for extensive research on the development of vaccines and treatments against the disease. Due to limited data on patients, the high infection rate of the disease, and the necessary regulations and testing delays for patient safety, these solutions may not be sufficient on their own for controlling the disease.

At the time of publication, there is no singular form of treatment for COVID-19 being endorsed by medical and government authorities. Based on the current form of treatments being tested, Zhai et al. (2020) have concluded that it will take a long time before any final testing reaches completion. However, data from interim results may be helpful or insightful for therapy in the meantime. There is reason to believe that treatments of related diseases will also be effective against COVID-19, particularly those for SARS and MERS. Because of the success of blood purification in treating SARS and MERS in the past, there is a high likelihood that it can also support COVID-19 rehabilitation (Yang et al. 2020a). Type 1 interferons have the same potential, having also been used to treat SARS and MERS. The publication of the data of China's utilization of related interferon strategies in COVID-19 treatment will further support this relation (Sallard et al. 2020).

Testing of treatments on actual cases of COVID-19 has actually been undertaken. Chloroquine and hydroxychloroquine have been tested at various doses in different countries with observed improvement in hematological parameters (Singh et al. 2020). These treatments have been proven effective in limiting the replication of the virus in vitro and observed to be safe in clinical trials (Cortegiani et al. 2020). However, only extensive high-quality clinical trials can prove that this treatment can safely be endorsed to the larger community.

\section{Model Development}

\section{Base Model}

The model that was formulated for the system dynamics analysis follows the SEIR model. Major feedback loops are graphically summarized in Fig. 1, while relevant variables are defined in Table 1. Four general states of susceptible (S), exposed (E) or carriers, infected (I), and recovery (R) were considered with different flow rates in between depending on the interaction of variables and latency under the Philippine condition. In loopwise, the model was divided into six primary loops, which may be classified into three types: focusing on (1) transmission to infection; (2) solutions such as social mobilization, public health care, and hospital care; and (3) sensitivity due to economic impact. Considering the known information about transmission of COVID-19 virus during the modeling, exposed and infected states can be either asymptomatic or symptomatic. Additionally, infected population is the only leverage point for solution implementation. For clarity, the operational definition of each loop is given in the succeeding sections.

\section{Feedback Loops}

\section{Rate of Transmission Loop}

The rate of transmission loop is the initial loop for infection coming from the infected rate under the factor of the current behavioral transmission rate to the susceptible population. The fractional susceptible population is resolved from deducting all the deaths that has happened in the system from the initial level of population.

\section{Rate of Infection Loop}

The rate of infection loop is the supporting loop that shows the transition of infected susceptible populations to an exposed 


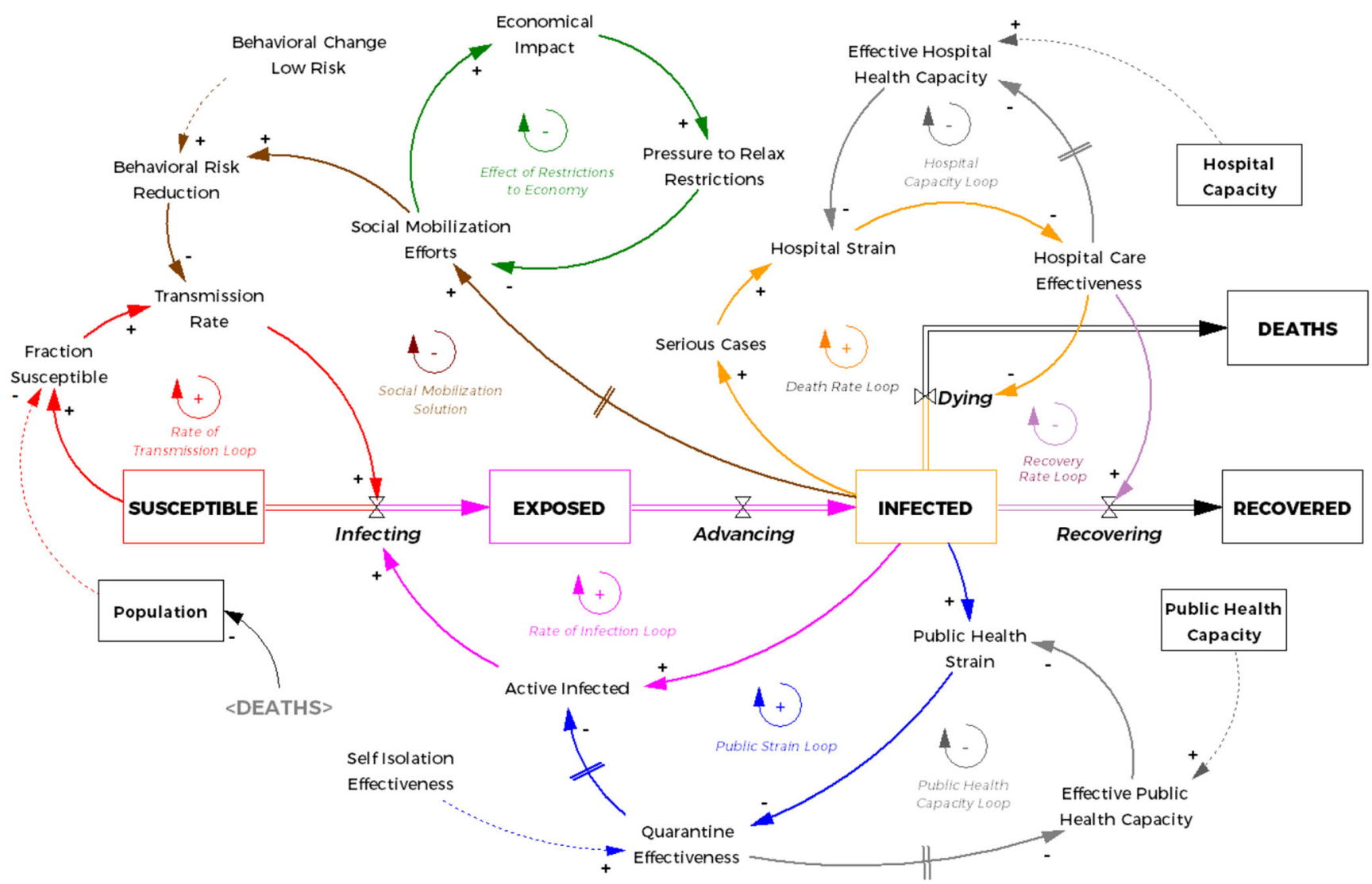

Fig. 1 Feedback loops for spread of pandemic disease with major stocks and flows

population or carrier of the virus. From being infected, people will be tagged as exposed which further advances as a known infected population which has a probability of transmitting the virus if not contained, quarantined, or monitored by the public health or hospital.

\section{Social Mobilization Loop}

The first solution loop aims to minimize the transmission rate via behavioral balancing measures as endorsed by the government; this is referred to as the social mobilization loop. This involves governmental mandated practices such as lockdowns, community quarantine, and private institution societal adjustments. This also taps on the behavioral practices such as social distancing, increased handwashing, and sterilization. These efforts can reduce the transmission rate to a particular extent.

\section{Public Strain and Public Health Capacity Loop}

The second solution loop focuses on the first-line measures of managing infected cases in testing, quarantining, contract tracing, etc. - measures both carried by the public health system and self-isolation to optimistically remove the possibility of the infected population to cause further transmission. Operationally, given that the case is less serious, the infected population will be endorsed to be isolated. Isolation may initially be carried out by the public health system and then progresses to home quarantine if the case improves and facilitating conditions are achieved. The system is capped both by the effectiveness decline from fatigue and depreciation due to resource usage.

\section{Death Rate Loop, Recovery Rate Loop, and Public Health and Hospital Capacity Loop}

The death and recovery loops, together with the public health and hospital capacity loop, are collectively classified as the third solution loop. This class holds the serious infected cases from spreading the virus to the susceptible population. This is considered to be the penultimate solution after social mobilization and public health measures. In this, serious cases are admitted in the hospitals whose effectiveness is dictated by the current capacity strain that it is withstanding. As the system continuously admits more patients up to the point of overloading, the system will collapse which negatively affects fatality rate. Conversely, when the strain experienced by the 
Table 1 Nomenclature

\begin{tabular}{|c|c|c|}
\hline Variables & Definition & Units \\
\hline \multicolumn{3}{|l|}{ Rate of transmission loop } \\
\hline Population & Population in the scope of the model & People \\
\hline Fraction susceptible & Fraction of initial population remaining susceptible in contracting COVID-19 & $\%$ \\
\hline Transmission rate & $\begin{array}{l}\text { Rate of transmission from non-isolated active infected to the } \\
\text { general susceptible population }\end{array}$ & $\% /$ day \\
\hline \multicolumn{3}{|l|}{ Rate of infection loop } \\
\hline Active infected & $\begin{array}{l}\text { People infected with COVID-19 that is actively outside from isolation, } \\
\text { quarantine, or monitoring by the public health or hospital }\end{array}$ & People \\
\hline \multicolumn{3}{|l|}{ Social mobilization loop } \\
\hline Social mobilization efforts & $\begin{array}{l}\text { Reduction in risk from governmental effort such as closure of establishment, } \\
\text { lockdowns, community quarantine, and the like }\end{array}$ & Dimensionless \\
\hline Behavioral risk reduction & $\begin{array}{l}\text { Risk reduction due to behavioral measures such as social distancing, } \\
\text { increased handwashing, sterilization, and the like }\end{array}$ & Dimensionless \\
\hline Behavioral change low risk & $\begin{array}{l}\text { Reduction in risk from societal adjustment for low-risk demographics } \\
\text { such as work-from-home setting, class suspension, and the like }\end{array}$ & Dimensionless \\
\hline \multicolumn{3}{|c|}{ Public strain loop and public health capacity loop } \\
\hline Public health capacity & $\begin{array}{l}\text { Ability of the public health system to manage infected cases in testing, } \\
\text { quarantining, contact tracing, etc. }\end{array}$ & Capacity/capita \\
\hline Public health strain & Burden of infected people relative to the effective public health capacity & Dimensionless \\
\hline Quarantine effectiveness & Fractional reduction in infections gained by quarantine in the public health system & Dimensionless \\
\hline Self-isolation effectiveness & $\begin{array}{l}\text { Fractional reduction in infections gained by isolation of infected person } \\
\text { outside the public health system }\end{array}$ & Dimensionless \\
\hline Effective public health capacity & $\begin{array}{l}\text { Effective capacity of the public health system, after adjusting for reduction } \\
\text { from fatigue and depreciation of resources }\end{array}$ & Capacity/capita \\
\hline \multicolumn{3}{|c|}{ Death rate loop, recovery rate loop, and public health and hospital capacity loop } \\
\hline Hospital capacity & $\begin{array}{l}\text { Ability of the hospitals to manage a number of serious cases given their } \\
\text { available resources such as beds, ventilators, doctors, nurses, etc. }\end{array}$ & Capacity/capita \\
\hline Serious case & Number of infected cases requiring hospitalization & Dimensionless \\
\hline Hospital strain & Strain in effective hospital health capacity due to overloading & Dimensionless \\
\hline Hospital care effectiveness & Effect of hospital strain to hospital efficacy which negatively affects fatality rate & Dimensionless \\
\hline Effective hospital health capacity & $\begin{array}{l}\text { Effective capacity of hospitals, after adjusting for reduction from } \\
\text { fatigue and depreciation of resources. }\end{array}$ & People \\
\hline Dying & Number of people dying after hospital care & People/day \\
\hline Recovering & Number of people recovering after hospital care & People/day \\
\hline \multicolumn{3}{|c|}{ Effect of restrictions to the economy loop } \\
\hline Economical impact & Aggregate effect on the economics of the country from social mobilization efforts & Dimensionless \\
\hline Pressure to relax restrictions & Change in the social mobilization effort due to sensitivity from economical impact & Dimensionless \\
\hline
\end{tabular}

public health and hospital sectors are more manageable, an improvement may be observed in the recovery rate.

\section{Effect of Restrictions to Economy}

A new introduction to the model is the decision-making in the overall effect of the mobilization efforts to the economy of the country. Operationally, as more closures and restrictions are imposed by the government from the social mobilization efforts, the economy is then significantly and increasingly hampered which increases the pressure to relax restrictions to operate. As this happens, it balances the decision by the government to the degree they will implement it.

\section{Simulation Results}

The SD model was implemented in the commercial software Vensim PLE, which may be acquired for free from Ventana Systems, Inc. Vensim PLE is a simulation software which primarily supports continuous simulation for SD by providing a graphical modeling interface for stock-and-flow and causal loop diagrams wherein text-based equations may be embedded.

A stock-and-flow diagram was developed to test the validity of the feedback relationships established in the previous section. Simple mathematical equations were used in the simulation model to express the relationships between variables, while parameters indicate the weight 
of these relationships. These equations (expressed in Vensim's programming language) are presented in the Appendix. The model's validity was demonstrated through testing model structure, consistency, and utility (Barlas 1992). Common response strategies to a pandemic were simulated in this section to ascertain their effectiveness in addressing the issue and minimizing its indirect consequences on healthcare capacity and the economy.

However, values obtained from results of SD simulations are not accurate for prediction or forecasting purposes. The results and graphs presented in this research cannot be used to accurately predict the number of case incidence and the timeline of the pandemic. Instead, SD is used to understand trends and behaviors of relevant problem variables over time as they are influenced by feedbacks within the system. Because system dynamics is a behavior-focused simulation technique, the sensitivity of behavior patterns, such as level and time, to parameter changes should be investigated to identify potential leverage points that would improve the system and the effect of uncertainty on the behaviors.

\section{Baseline Behavior}

The SD model is initially used to generate a set of baseline results. The baseline behavior is presented in terms of key variables, which are selected based on their ability to describe the well-being of a population. Said key variables are infection, death, hospital strain, and public health strain (Fig. 2a-c). Given that the core problem is disease transmission, the importance of variables related to the status of health (i.e., sick and active infected) is important. However, as the behavior of hospital strain (Fig. 2b) and public health strain (Fig. 2c) follows that of the sick, the analysis will focus on deaths, hospital strain, and public health strain in particular.

\section{Public Health Capacity}

Hospital and public policy strain represent the risk, resource consumption, and general socioeconomic impact of handling a pandemic. As such, policies to increase or make efficient use of these resources are an obvious solution to reduce strain on these sectors. Two means of increasing public health capacity (PHC) are tested: (1) increasing in anticipation of the pandemic $(+50 \%$ PHC,$+100 \%$ PHC,$+50 \%$ PHC + PIE) and (2) increasing PHC in the middle of the pandemic (RAMP PHC). Increasing PHC in anticipation of the pandemic will evidently result in lower capacity strain (Fig. 3b-c), since PHC cannot be increased at once. This significantly lowers the volume of active infected and deaths (Fig. 3a). Moreover, increasing $\mathrm{PHC}$ and increasing potential isolation effectiveness (PIE) simultaneously $(+50 \%$ PHC + PIE) can achieve a reduction in active infected and deaths equivalent to that of increasing PHC by a higher percentage $(+100 \%$ PHC). It can also be noted that increasing hospital capacity will have similar improvements. However, as PHC addresses public health strain while hospital capacity does not, the reduction in active cases and deaths will be less.

\section{Behavior Risk Reduction}

Behavior risk reduction (BRR) reduces the transmission rate of the disease. When implemented alongside quarantine policies, BRR can significantly widen and flatten the number of active cases and lower the number of deaths. The wider active cases curve under BRR (Q. Pol + 80\% BRR - 50\% BCLR, Q. Pol $+80 \%$ BRR $-100 \%$ BCLR, Q. Pol $+80 \%$ BRR) is attributed to the delayed onset of policies that are triggered by the volume of active cases and sick. BRR is tested with different rates of behavioral change low risk (BCLR), which represents cases where quarantine is relaxed for low risk. From the

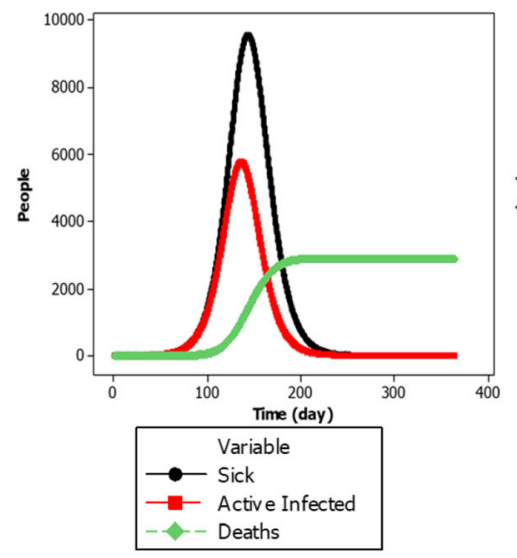

(a)

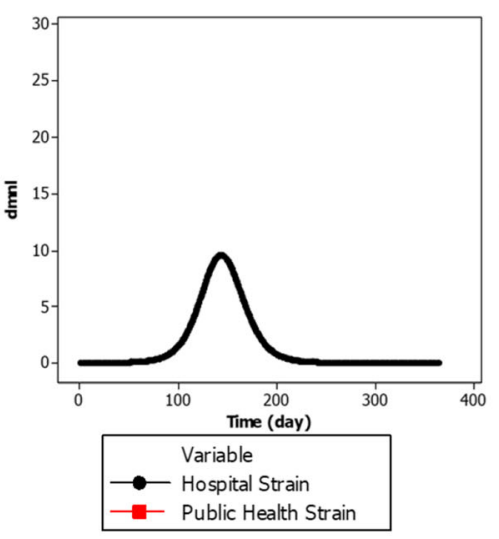

(b)

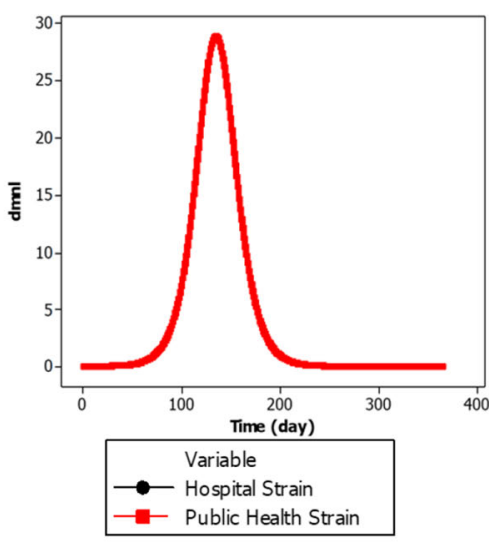

(c)

Fig. 2 Baseline behavior for (a) population size of infected and dead, (b) hospital strain, and (c) public health strain 


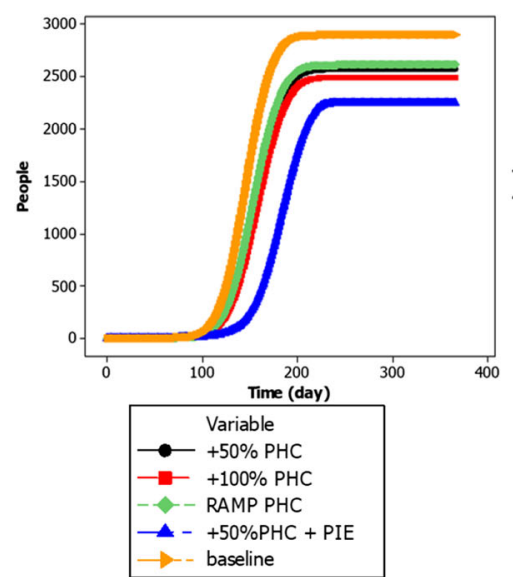

(a)

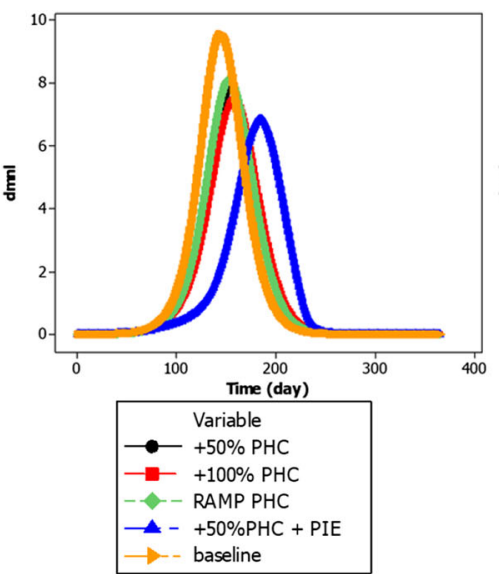

(b)

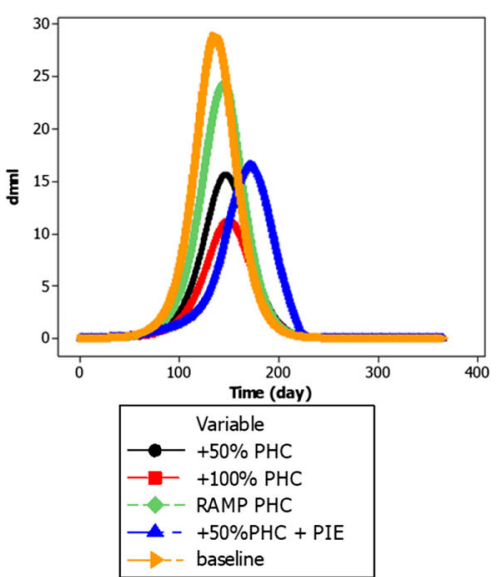

(c)

Fig. 3 Behavior under public health capacity policy for (a) deaths, (b) hospital strain, and (c) public health strain

results (Fig. 4), it is evident that implementing the policy in full will have significantly lower active cases, deaths, and strain. Furthermore, combining behavior risk reduction strategies with self-isolation is found to be a more effective strategy for reducing overall number of cases, deaths, and strains on public health and hospitals because it further lowers the probability of exposure and transmission. However, this is challenging to achieve because it is an individual-based solution, which would necessitate or depend heavily on the accessibility and accuracy of testing and efficiency of contact tracing.

\section{Economic Sensitivity}

In relation to BRR, changing BCLR is intended to preserve the health of the economy. Another means of reducing economic impact is through policies for economic sensitivity, which changes the rate of risk reduction according to previous social mobilization efforts. The intuition behind this policy is that it adapts to the current state of infection and also gives consideration for economic objectives. The results of this policy are contrasted with the results of BRR (Fig. 5). Economic sensitivity reduces the width of the active infected curve but also maintains a significantly lower peak infection than the baseline.

\section{Gradual Enhanced Community Quarantine Lifting Policy}

Given that implementing BRR in full still results in lower levels of active infected and deaths, a policy for gradual lifting rather than complete lifting after an extended period was also considered. The gradual enhanced community quarantine (ECQ) lifting is implemented by introducing a RAMP function to bring BRR from 80 to $0 \%$, beginning at a certain period. Likewise with economic sensitivity, this can also be applied to preserve economic health, albeit with less volatility

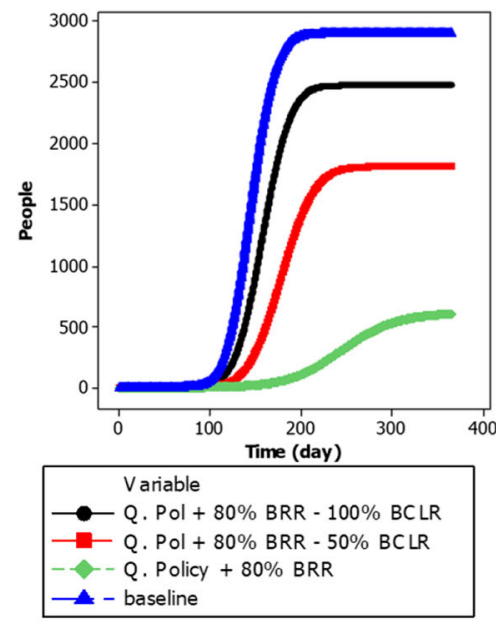

(a)

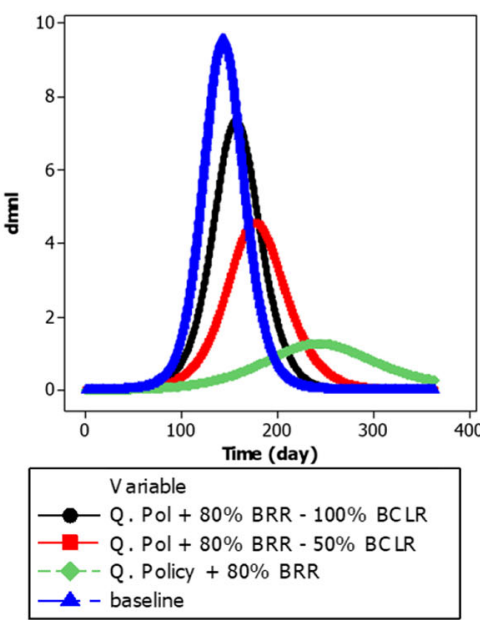

(b)

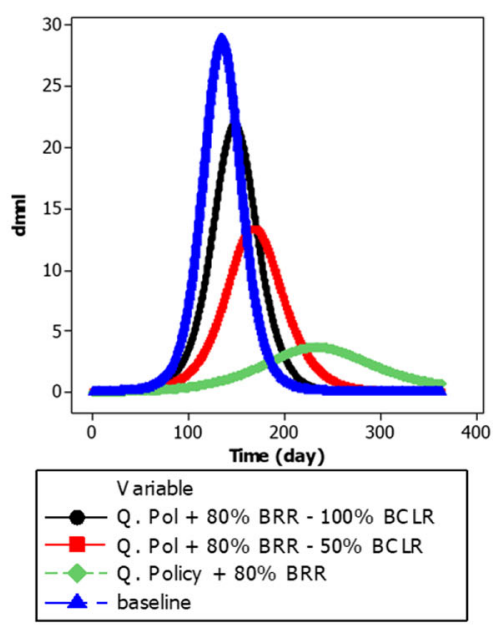

(c)

Fig. 4 Behavior under behavior risk reduction policy for (a) deaths, (b) hospital strain, and (c) public health strain 


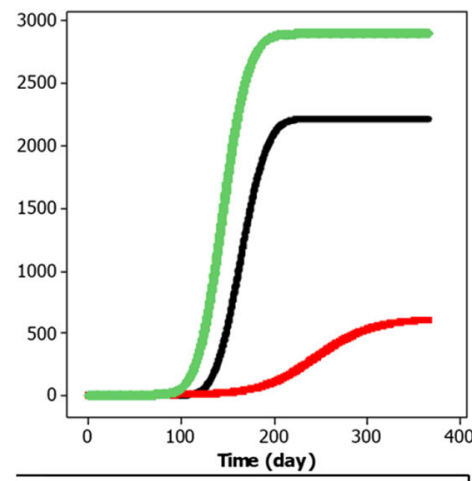

V ariable

$50 \%$ BRR + Inc. Economy Sensitiv

Q. Pol $+80 \%$ BRR

baseline

(a)

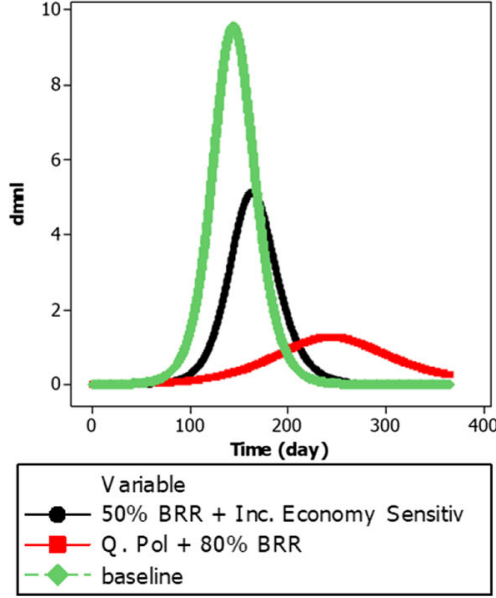

(b)

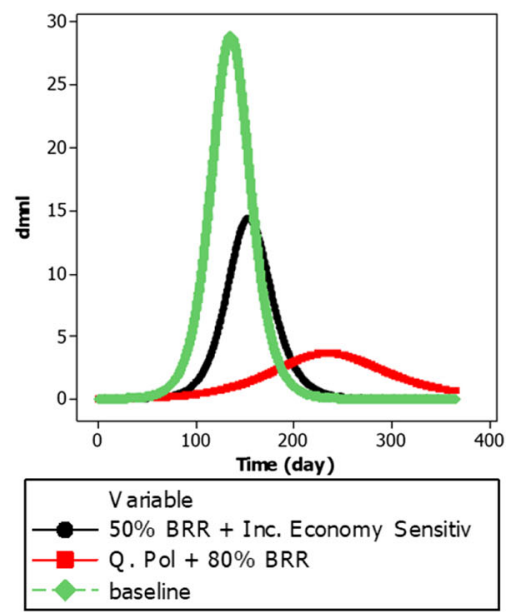

(c)

Fig. 5 Behavior under economic sensitivity policy for (a) deaths, (b) hospital strain, and (c) public health strain

and a larger time delay. The results show that a certain threshold (1 year ecq lift @ 250, 1 year ecq lift @ 200) is necessary to achieve results equivalent to implementing BRR in full. However, even lowering the threshold (1 year ecq lift @ 120) will achieve a lower peak when compared to the economic sensitivity policy (Fig. 6).

\section{Policy Insights/Recommendations}

The SD model allows various strategies for pandemic preparedness and response to be simulated. The effectiveness of each solution was evaluated based on the number of people infected and deaths within a population, the duration a population suffers from the pandemic, and the strain experienced by the public health sector, hospitals, and the economy. Policy insights and recommendations are summarized as follows:
- Delaying the onset of the disease outbreak through preventive and precautionary measures can allow the government more time to prepare the necessary resources and measures to control the virus. This results in a wider curve, which prolongs the duration of the disease within the population but reduces the risk of overloading the capacities of private and public healthcare facilities by reducing and delaying the peak of the pandemic. Intuitively, SD simulation modeling confirms that delayed response just as the pandemic is beginning would severely worsen the effect and duration of the pandemic within a community.

- Pandemic preparedness demonstrated by having high existing capacities for health services facilities, personnel, and supplies will go a long way in reducing mortality rates and hospital strain. However, in countries that cannot afford to have excess capacities readily installed in anticipation of pandemics, not much difference was observed between a

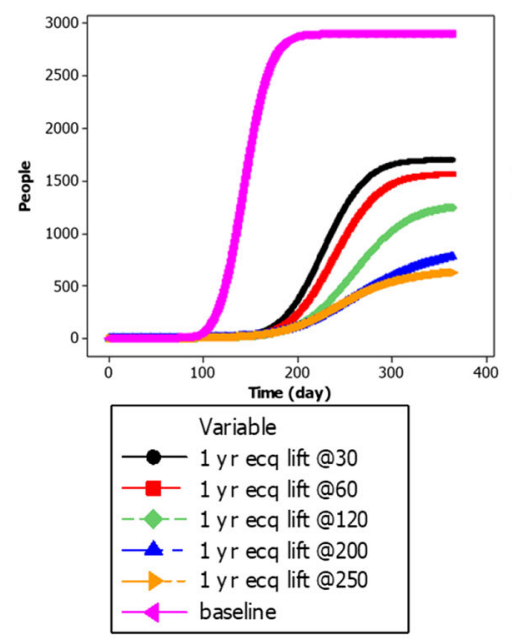

(a)

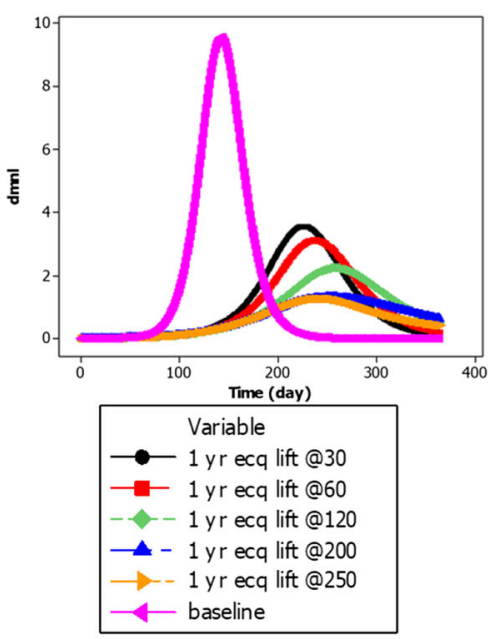

(b)

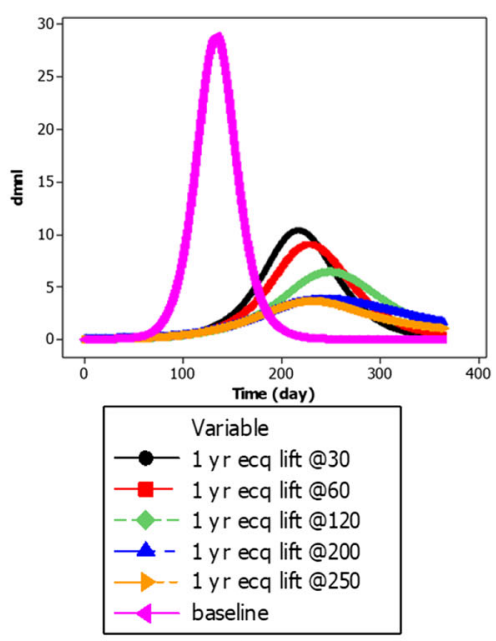

(c)

Fig. 6 Behavior under gradual ECQ lifting policy for (a) deaths, (b) hospital strain, and (c) public health strain 
gradual increase in hospital capacity and an instant dramatic increase in terms of hospital strain and number of deaths. This is given that the delay in responding to the hospital capacity problem is not too long.

- The most effective interventions revolved around solutions that decrease exposure and transmissions, such as through self-isolation, social distancing, and community quarantines. However, blindly implementing excessively stringent policies would not be beneficial if it does not properly target the problem and at the same time incurs the community significant economic losses. Hence, effective and mass testing protocols and contact tracing will significantly improve the system because it allows selfisolation to be efficiently implemented. Technological innovations that support detection would also be beneficial.

- Nonetheless, caving into economic pressures and relaxing quarantine pressures too early will invalidate any progress to slow down and temper the pandemic. This only delays the peak of the infection but does nothing to significantly reduce the number of infections and may even result in a "second wave." For instance, allowing low-risk people to return to business-as-usual can increase the spread of the virus. It is important to emphasize that lives and livelihoods cannot be simultaneously saved and be the focus of the current problem. Prioritizing health will have a better impact on the system as a whole compared to trying to solve both problems at the same time, as it will allow the system to resume economic activities as soon as the health problem is secured.

- The "second wave" after lifting of community quarantine may be avoided through effective individual and personal risk reduction strategies, such as social distancing and wearing of proper personal protective equipment.

- Lifting the community quarantines gradually and indefinitely implementing the quarantine have similar effects on the pandemic's behavior, especially if the gradual relaxation is slowly calibrated based on industry characteristics and risk. However, gradual lifting is more cost-effective and results in less strain on the economy because it allows certain businesses to resume activities, instead of a complete standstill. Furthermore, after a certain duration, extending the quarantine will no longer improve the infection rate but makes it increasingly more difficult for the community to recover from the pandemic.

\section{Conclusions and Future Directions}

This study was undertaken to develop and demonstrate an efficient methodology for policy development in response to the COVID-19 pandemic. It recognizes that adherence to rigid mathematical representations makes it difficult to capture important aspects of policy deployment, particularly human response and policy-maker control loops. As such, it relaxes numerical exactness in favor of a holistic and descriptive model. The system dynamics framework makes it possible to simulate the unprecedented situation, in spite of limited data, through an understanding of the elements involved and their relationships with the problem variable. More importantly, this model can be used to develop and simulate the policies to control and ultimately stop infection.

In the context of COVID-19, social mobilization is a form of human response with critical impact on infection. The model was able to show that these efforts are critical to the success of other policies, by stopping the problem at the earliest point of infection. Policy-maker control loops are integrated in the decisions to expand public health capacity, and the geographical and demographical scope of quarantine. The loop exists in the feedback between current infection levels and these decisions.

The model was able to produce generalizable insights for policy response. In summary, (1) social mobilization has been identified as a means to delay the onset of the pandemic, resulting in an infection curve that is wider with a lower peak. This contributes to lowering the strain on quarantine and hospital capacity. (2) Increasing public health capacity is necessary but does not significantly change the dynamic behavior of infection. The results showed that the earlier the additional capacity could be introduced, the less the capacity strain in the long term. (3) Partial quarantine lifts in any form will prolong the duration of the pandemic without significantly reducing peak infection.

The current model can be adapted for the situations of different countries. It serves as a base model with only the most common and recent pandemic responses. To represent situations more specifically, sub-systems can be added to represent more stakeholders or policy implementation points, such as delving into testing protocols and different economic sectors. Moreover, parameter tuning would need to be employed, particularly in the areas of initial population and public health capacity, to make the values and timing of the dynamic variables more consistent with the real system. Furthermore, as COVID-19 vaccines start entering final stages of testing and begin distribution, extensions of this modeling work can explore the long-term implications of this development. Several factors will still influence the impact of COVID-19 vaccines on the pandemic, such as how fast the vaccines are approved for national use, manufactured, and distributed, the number of people who decide to get vaccinated, the effectiveness of the vaccine, how long after vaccination is immunity built, and whether the vaccine offers long-term protection among some.

Overall, the methodology demonstrated in this study shows how situations without prior data can be modeled through the analysis of relationships. Although there are limits to what can be inferred about the future, just the identification of the 
critical elements of a problem can provide policy-makers with ample room for solution development.

\section{Compliance with Ethical Standards}

Conflict of Interest The authors declare that they have no conflicts of interest.

\section{Appendix. Model Code}

\section{A. Stocks}

Deaths $=$ INTEG(Dying,0)

Exposed= INTEG (Infecting-Emerging,0)

Infectious Asymptomatic= INTEG (Emerging-Onset,0)

Infectious Symptomatic= INTEG (Onset-Advancing,0)

Recovered $=$ INTEG $($ Recovering, 0$)$

Sick= INTEG (Advancing-Dying-Recovering,0)

Susceptible= INTEG (-Infecting,Initial Population)

Total Infections from Overseas= INTEG (Infections from overseas, 0 )

\section{B. Flows}

Advancing=Infectious Symptomatic/Time to Advance Dying=Sick*Fatality Rate/Infection Duration

Emerging=Exposed/Time to Infectious

Infecting $=($ Active Infected $*$ Transmission Rate $)+$ Infections from overseas

Infections from overseas $=\mathrm{N}$ Imported Infections*PULSE(Import Time,TIME STEP)/TIME STEP

Onset=Infectious Asymptomatic/Time to Onset

Recovering=Sick/Infection Duration*(1-Fatality Rate)

\section{Auxiliary Variables}

Active Infected $=(($ Infectious Symptomatic $) *(1-$ Relative Isolation Effectiveness Pre Symptoms) +(Infectious Asymptomatic)*(1-Self Isolation Effectiveness/4))*(1Quarantine Effectiveness*"Q. Policy Switch")

Economic Impact of Quarantine and Restrictions=Social Mobilization Efforts+Quarantine efforts

Effect of impact to risk behavior= $(1-1 /(1+$ Pressure to relax restrictions ${ }^{\wedge}$ Sensitivity of Economy to Risk Behavior))

Fatality Rate $=$ Untreated Fatality Rate+(Treated Fatality Rate-Untreated Fatality Rate)/(1+Hospital Strain^Hospital Capacity Sensitivity)

Hospital Strain=Serious Cases/Hospital Capacity

Infected Case Load=Exposed+Infectious Asymptomatic+ Infectious Symptomatic

Initial Uncontrolled Transmission Rate=R0/Infection Duration

Observed Active Cases $=$ Sick+Infectious Symptomatic
Pressure to relax restrictions=Economic Impact of Quarantine and Restrictions/5

Public Health Strain=Infected Case Load/Public Health Capacity

Quarantine Effectiveness=Quarantine efforts $/(1+$ Public Health Strain^Public Health Capacity Sensitivity)

Quarantine efforts $=($ SMOOTH3(STEP(Potential Isolation Effectiveness,Import Time),Isolation Reaction Time))*Cases per Capita

Relative Behavior Risk = (SMOOTH3( 1 STEP((Behavioral Risk Reduction/4)*Behavior Change for Low risk*((1+Social Mobilization Efforts*"Social Pol. Switch")/(1+Effect of impact to risk behavior*"Social Pol. Switch")),Import Time),Behavior Reaction Time))

Relative Contact Density $=1 /(1+$ Contact Density Decline*(1-Fraction Susceptible))

Serious Cases=Sick*Fraction Requiring Hospitalization

Social Mobilization Efforts=Cases per Capita/(Behavior Change Threshold+Fatigue Threshold)

Total Population=Initial Population-Deaths

Transmission Rate=Initial Uncontrolled Transmission Rate*Relative Behavior Risk*Fraction Susceptible *Relative Contact Density

\section{References}

Alamerew YA, Brissaud D (2020) Modelling reverse supply chain through system dynamics for realizing the transition towards the circular economy: a case study on electric vehicle batteries. J Clean Prod 254:120025

Araz OM (2013) Integrating complex system dynamics of pandemic influenza with a multi-criteria decision making model for evaluating public health strategies. J Syst Sci Syst Eng 22(3):319-339. https:// doi.org/10.1007/s11518-013-5220-y

Barlas Y (1992) Comments on "On the very idea of a system dynamics model of Kuhnian science". Syst Dyn Rev 8(1):43-47. https://doi. org/10.1002/sdr.4260080105

Brooks SK, Webster RK, Smith LE, Woodland L, Wessely S, Greenberg N, Rubin GJ (2020) The psychological impact of quarantine and how to reduce it: rapid review of the evidence. Lancet 395:912-920

Cao Y, Zhao Y, Wen L, Li Y, Wang S, Liu Y et al (2019) System dynamics simulation for $\mathrm{CO} 2$ emission mitigation in green electric-coal supply chain. J Clean Prod 232:759-773

Chevance A, Gourion D, Hoertel N, Llorca PM, Thomas P, Bocher R, ... Masson M (2020) Ensuring mental health care during the SARSCoV-2 epidemic in France: a narrative review. L'Encéphale

Chintalapudi N, Battineni G, Amenta F (2020) COVID-19 disease outbreak forecasting of registered and recovered cases after sixty day lockdown in Italy: a data driven model approach. J Microbiol Immunol Infect 53:396-403

Cortegiani A, Ingoglia G, Ippolito M, Giarratano A, Einav S (2020) A systematic review on the efficacy and safety of chloroquine for the treatment of COVID-19. J Crit Care

Culaba AB, Lim Ching P, San Juan JL, Philip Mayol A, Sybingco E, Ubando AT (2019) A dynamic sustainability assessment of algal biorefineries for biofuel production. 2019 IEEE $11^{\text {th }}$ International Conference on Humanoid, Nanotechnology, Information Technology, Communication and Control, Environment, and 
Management ( HNICEM ). https://doi.org/10.1109/hnicem48295. 2019.9072721

Currie CS, Fowler JW, Kotiadis K, Monks T, Onggo BS, Robertson DA, Tako AA (2020) How simulation modelling can help reduce the impact of COVID-19. Journal of Simulation 14(2):83-97. https:// doi.org/10.1080/17477778.2020.1751570

Dénes A, Gumel AB (2019) Modeling the impact of quarantine during an outbreak of Ebola virus disease. Infectious Disease Modelling 4:1227

Deng S, Ji J, Wen G, Xu H (2020) Delay-induced novel dynamics in a hexagonal centrifugal governor system. International Journal of Non-Linear Mechanics 121:103465

Doyle JK, Ford DN (1998) Mental models concepts for system dynamics research. System dynamics review: the journal of the System Dynamics Society 14(1):3-29

Ejima K, Nishiura H (2018) Real-time quantification of the nextgeneration matrix and age-dependent forecasting of pandemic influenza H1N1 2009 in Japan. Ann Epidemiol 28(5):301-308

Espinoza A, Bautista S, Narváez P, Alfaro M, Camargo M (2017) Sustainability assessment to support governmental biodiesel policy in Colombia: a system dynamics model. J Clean Prod 141:11451163. https://doi.org/10.1016/j.jclepro.2016.09.168

Fontes CHDO, Freires FGM (2018) Sustainable and renewable energy supply chain: a system dynamics overview. Renew Sust Energ Rev $82: 247-259$

Forrester JW (1994) System dynamics, systems thinking, and soft OR. Syst Dyn Rev 10(2-3):245-256

Germann TC, Gao H, Gambhir M, Plummer A, Biggerstaff M, Reed C, Uzicanin A (2019) School dismissal as a pandemic influenza response: when, where and for how long? Epidemics 28:100348

Giovannelli J, Loury P, Lainé M, Spaccaferri G, Hubert B, Chaud P (2015) Forecasts of health care utilization related to pandemic a (H1N1) 2009 influenza in the Nord-Pas-de-Calais region, France. Public Health 129(5):493-500

Gupta SS, Gupta SK (2020) Social mobilization for cholera prevention \& control in India: building on the existing framework. Vaccine 38: A181-A183

Hart OE, Halden RU (2020) Computational analysis of SARS-CoV-2/ COVID-19 surveillance by wastewater-based epidemiology locally and globally: feasibility, economy, opportunities and challenges. Sci Total Environ 730:138875

Holmes EA, O'Connor RC, Perry VH, Tracey I, Wessely S, Arseneault L et al (2020) Multidisciplinary research priorities for the COVID-19 pandemic: a call for action for mental health science. Lancet Psychiatry

Ibarra-Vega D (2020) Lockdown, one, two, none, or smart. Modeling containing COVID-19 infection. A conceptual model. Sci Total Environ 730:138917. https://doi.org/10.1016/j.scitotenv.2020. 138917

Kuo TC, Lin SH, Tseng ML, Chiu AS, Hsu CW (2019) Biofuels for vehicles in Taiwan: using system dynamics modeling to evaluate government subsidy policies. Resour Conserv Recycl 145:31-39

Le TT, Andreadakis Z, Kumar A, Roman RG, Tollefsen S, Saville M, Mayhew S (2020) The COVID-19 vaccine development landscape. Nat Rev Drug Discov 19:667-668

Lei C, Zhou J (2020) Long-time dynamics of a reaction-diffusion system with negative feedback and inhibition. Appl Math Lett 107:106475

Li MY, Muldowney JS (1995) Global stability for the SEIR model in epidemiology. Math Biosci 125(2):155-164. https://doi.org/10. 1016/0025-5564(95)92756-5

Pappa S, Ntella V, Giannakas T, Giannakoulis VG, Papoutsi E, Katsaounou P (2020) Prevalence of depression, anxiety, and insomnia among healthcare workers during the COVID-19 pandemic: a systematic review and meta-analysis (in press). Brain Behav Immun

Safarishahrbijari A, Teyhouee A, Waldner C, Liu J, Osgood ND (2017) Predictive accuracy of particle filtering in dynamic models supporting outbreak projections. BMC Infect Dis 17(1):1-12. https://doi.org/10.1186/s12879-017-2726-9

Sallard E, Lescure FX, Yazdanpanah Y, Mentre F, Peiffer-Smadja N, Florence ADER et al (2020) Type 1 interferons as a potential treatment against COVID-19. Antivir Res 104791

Santos-Preciado J, Franco-Paredes C, Hernandez-Flores I, Tellez I, Del Rio C, Tapia-Conyer R (2009) What have we learned from the novel influenza A (H1N1) pandemic in 2009 for strengthening pandemic influenza preparedness? Arch Med Res 40(8):673-676

Simpson RJ, Katsanis E (2020) The immunological case for staying active during the COVID-19 pandemic. Brain Behav Immun 87:6-7

Singh AK, Singh A, Shaikh A, Singh R, Misra A (2020) Chloroquine and hydroxychloroquine in the treatment of COVID-19 with or without diabetes: a systematic search and a narrative review with a special reference to India and other developing countries. Diabetes Metab Syndr Clin Res Rev

Su K, Xu L, Li G, Ruan X, Li X, Deng P et al (2019) Forecasting influenza activity using self-adaptive AI model and multi-source data in Chongqing, China. EbioMedicine 47:284-292

Sun L, DePuy GW, Evans GW (2014) Multi-objective optimization models for patient allocation during a pandemic influenza outbreak. Comput Oper Res 51:350-359

Sy C, Bernardo E, Miguel A, San Juan JL, Mayol AP, Ching PM, Culaba A, Ubando A, Mutuc JE (2020) Policy development for pandemic response using system dynamics: a case study on COVID-19. Process Integration and Optimization for Sustainability 4:497-501. https://doi.org/10.1007/s41660-020-00130-x

Tang B, Xia F, Tang S, Bragazzi NL, Li Q, Sun X et al (2020) The effectiveness of quarantine and isolation determine the trend of the COVID-19 epidemics in the final phase of the current outbreak in China. Int J Infect Dis

Thiel D, Le Hoa Vo T, Hovelaque V (2014) Forecasts impacts on sanitary risk during a crisis: a case study. Int J Logist Manag 25(2):358-378. https://doi.org/10.1108/IJLM-04-2012-0028

Towers S, Geisse KV, Zheng Y, Feng Z (2011) Antiviral treatment for pandemic influenza: assessing potential repercussions using a seasonally forced SIR model. J Theor Biol 289:259-268

Tuckwell HC, Toubiana L (2007) Dynamical modeling of viral spread in spatially distributed populations: stochastic origins of oscillations and density dependence. BioSystems 90(2):546-559. https://doi. org/10.1016/j.biosystems.2006.12.006

Vo TLH, Thiel D (2011) Economic simulation of a poultry supply chain facing a sanitary crisis. Br Food J 113(8):1011-1030. https://doi.org/ 10.1108/00070701111153760

Wang J, Lai JY (2020) Exploring innovation diffusion of two-sided mobile payment platforms: a system dynamics approach. Technol Forecast Soc Chang 157:120088

Wang J, Liao Y, Wang X, Li Y, Jiang D, He J et al (2020) Incidence of novel coronavirus (2019-nCoV) infection among people under home quarantine in Shenzhen, China. Travel Med Infect Dis 37: 101660

Webster RK, Brooks SK, Smith LE, Woodland L, Wessely S, Rubin GJ (2020) How to improve adherence with quarantine: rapid review of the evidence. Public Health 182:163-169

Wenzel M, Stanske S, Lieberman MB (2020) Strategic responses to crisis. Strateg Manag J. https://doi.org/10.1002/smj.3161

Westerhoff L, Sheppard SR, Iype DM, Cote S, Salter J (2018) Social mobilization on climate change and energy: an evaluation of research projects in British Columbia, Canada. Energy Res Soc Sci 46:368-380

Yang XH, Sun RH, Zhao MY, Chen EZ, Liu J, Wang HL et al (2020a) Expert recommendations on blood purification treatment protocol for patients with severe COVID-19: recommendation and consensus. Chronic Diseases and Translational Medicine 6:106-114 
Yang Y, Zhang H, Chen X (2020b) Coronavirus pandemic and tourism: dynamic stochastic general equilibrium modeling of infectious disease outbreak. Ann Tour Res 83:102913

Zhai P, Ding Y, Wu X, Long J, Zhong Y, Li Y (2020) The epidemiology, diagnosis and treatment of COVID-19. Int J Antimicrob Agents 55: 105955

Zhang SX, Liu J, Jahanshahi AA, Nawaser K, Yousefi A, Li J, Sun S (2020) At the height of the storm: healthcare staff's health conditions and job satisfaction and their associated predictors during the epidemic peak of COVID-19 (in press). Brain Behav Immun
Zhao R, Zhou X, Jin Q, Wang Y, Liu C (2017) Enterprises' compliance with government carbon reduction labelling policy using a system dynamics approach. J Clean Prod 163:303-319

Zhu S, Wu Y, Zhu CY, Hong WC, Yu ZX, Chen ZK et al (2020) The immediate mental health impacts of the COVID-19 pandemic among people with or without quarantine managements. Brain Behav Immun

Publisher's Note Springer Nature remains neutral with regard to jurisdictional claims in published maps and institutional affiliations. 